\title{
MHY218-induced apoptotic cell death is enhanced by the inhibition of autophagy in AGS human gastric cancer cells
}

\author{
PYOUNG RAK CHOI ${ }^{2 *}$, YONG JUNG KANG ${ }^{1 *}$, BOKYUNG SUNG ${ }^{1}$, JAE HYUN KIM ${ }^{2}$, HYUNG RYONG MOON ${ }^{1}$, \\ HAE YOUNG CHUNG ${ }^{1}$, SUNG EUN KIM ${ }^{2}$, MOO IN PARK ${ }^{2}$, SEUN JA PARK ${ }^{2}$ and NAM DEUK KIM ${ }^{1}$ \\ ${ }^{1}$ College of Pharmacy, Molecular Inflammation Research Center for Aging Intervention (MRCA), Pusan National University, \\ Busan 609-735; ${ }^{2}$ Department of Gastroenterology, Kosin University Gospel Hospital, Busan 602-702, Republic of Korea
}

Received April 9, 2015; Accepted May 18, 2015

DOI: 10.3892/ijo.2015.3031

\begin{abstract}
We previously reported the anticancer effects of MHY218, which is a hydroxamic acid derivative, in HCT116 human colon cancer cells. In the present study, the involvement of autophagy in the MHY218-induced apoptotic cell death of AGS human gastric cancer cells was investigated. MHY218 treatment induced growth inhibition and apoptotic cell death in a concentration- and time-dependent manner. The induction of apoptosis was confirmed by observations of decreased viability, DNA fragmentation, and an increase in late apoptosis and sub-G1 DNA, which were detected with a flow cytometric analysis. Western blot analyses showed that MHY218 treatment resulted in decreased protein levels of procaspase-8, -9, and -3; cleavage of poly(ADP-ribose) polymerase (PARP); and alterations in the ratio of $\mathrm{Bax} / \mathrm{Bcl}-2$ protein expression. Apoptosis induced by MHY218 was involved in the activation of caspase-8, -9 , and -3 , and it was blocked by the addition of Z-VAD-FMK, a pan-caspase inhibitor. In addition, autophagyinducing effects of MHY218 were indicated by cytoplasmic vacuolation, the accumulation of acidic vesicular organelles, the appearance of green fluorescent protein-light-chain 3 (LC3) punctate dots, and increased levels of Beclin-1 and LC3-II protein expression. Pretreatment with the autophagy inhibitors LY294002, 3-methyladenine, chloroquine, and bafilomycin A1 enhanced the induction of apoptosis by MHY218, and this was accompanied by an increase in PARP cleavage. Taken together, these results provide new insights into the role of MHY218 as a potential antitumor agent. The
\end{abstract}

Correspondence to: Dr Nam Deuk Kim, Department of Pharmacy, College of Pharmacy, Pusan National University, Busan 609-735, Republic of Korea

E-mail: nadkim@pusan.ac.kr

Dr Seun Ja Park, Department of Gastroenterology, Kosin University Gospel Hospital, Busan 602-702, Republic of Korea

E-mail: parksj6406@daum.net

*Contributed equally

Key words: MHY218, gastric cancer cells, apoptosis, autophagy combination of MHY218 with an autophagy inhibitor might be a useful candidate for the chemoprevention and/or treatment of gastric cancer.

\section{Introduction}

According to GLOBOCAN 2012, an estimated 14.1 million new cancer cases and 8.2 million cancer-related deaths occurred in 2012 compared with 12.7 million and 7.6 million, respectively, in 2008. Gastric cancer (GC) is the fifth most common cancer (0.9 million, 6.8\%) in the world after lung, breast, colorectal, and prostate cancers and the second leading cause of cancer death worldwide ( 0.7 million, $8.8 \%)$ after lung cancer. Historically, GC is one of the major cancers in Asian countries, such as Korea and Japan. Although the mortality and incidence of GC have decreased worldwide, 31,269 new GC cases were reported in 2012, accounting for $14.2 \%$ of all cancer occurrences $(219,520$ case/year; data available at http://www.cancerresearchuk.org/cancer-info/ cancerstats/world/incidence/). The most effective treatment for localized GC is surgery, but, even after curative resection, recurrence is noted in more than half of the cases with advancedstage disease. Moreover, although the effective treatments that are available have increased the survival rates of GC patients during the last three decades, the prognoses of these patients remain relatively poor, with a 5-year survival rate of $63.1 \%$ during 2004-2008 (http://www.cancer.go.kr/mbs/cancer/ subview.jsp?id=cancer_040101000000). Due to the high recurrence rate after resection surgery, patients with GC are treated with adjuvant chemotherapy (including cisplatin, 5-fluorouracil, capecitabine, oxaliplatin, mitomycin $\mathrm{C}$, and etoposide) and/or radiation therapy. However, the efficacies of these adjuvant therapies are limited because of the side effects, such as nausea, vomiting, the high risk of infection, and diarrhea (1). Thus, the development of novel anticancer agents for patients with GC and effective treatment strategies are urgently required to increase their efficacy.

Autophagy is a process that involves the cellular degradation of unnecessary or dysfunctional cellular components and the maintenance of cellular homeostasis. During autophagy, parts of the cytoplasm and cellular organelles are engulfed within double-membraned autophagosomes, and the autophagosomes fuse with lysosomes to form autolysosomes. This 
results in the degradation of the sequestered cargo by lysosomal hydrolytic enzymes. The degradation is followed by the generation of cellular building blocks for biosynthesis and energy production. Because autophagy reuses cellular components when dealing with cellular stress by clearing damaged proteins, organelles, or pathogens and provides the resources for biosynthesis (e.g., adenosine triphosphate or anabolic building blocks) during starvation, this process was initially recognized as a mechanism that was cytoprotective against environmental stress. Conversely, increasing amounts of recent evidence have revealed that autophagy can also culminate in cell death, which is designated as type- 2 programmed cell death or autophagic cell death (2).

The essential role of autophagy in cancer is not clearly understood; however, its role in cell death is conflicting depending on the type of tumor, the stage of tumorigenesis, and the nature and extent of the stimuli (3-5). It is generally believed that these two self-destructive processes, autophagy and apoptosis, often occur in the same cell and that autophagy mostly precedes apoptosis (6,7). Furthermore, some chemotherapeutics that are known to trigger apoptosis also induce autophagy (8). In addition, emerging evidence indicates that the inhibition of autophagy appears to enhance the sensitivity of cancer cells towards anticancer drugs (9). Based on the aforementioned findings, it would be helpful to develop a new anticancer agent that simultaneously induces both autophagy and apoptosis. Thus, the elucidation of the role of autophagy and the determination of whether it is induced by agents in the response of tumor cells to that agent will provide therapeutic advantages by manipulating the autophagic process.

The anticancer effects of the synthetic hydroxamic acid derivative MHY218 [ $\mathrm{N}^{1}$-hydroxy- $\mathrm{N}^{8}$-(4-phenoxyphenol) octanedianide], was first reported by Jeon and colleagues (10). It was found to cause apoptosis in SKOV-3 ovarian cancer cells. This histone deacetylase (HDAC) inhibitor exhibits growth inhibitory effects in several tumor cell lines, including ovarian, breast, and colon cancer cells (10-12). Two studies in animals have shown that MHY218 has antineoplastic activities against tamoxifen-resistant breast cancer and ovarian cancer $(10,11)$. The anticancer mechanisms of MHY218 are still under investigation, and few studies have reported its effects on cell death. It has been shown to induce the pro-apoptotic Bax protein, inhibit the anti-apoptotic Bcl-2 protein (10-12), and suppress the nuclear translocation of nuclear factor- $\kappa \mathrm{B}$ $(\mathrm{NF}-\kappa \mathrm{B})(12)$. Thus, modulations of the NF- $\mathrm{B}$-regulated genes such as cyclooxygenase-2, matrix metalloproteinase-9, 5-lipoxygenase (12), and HDACs $(10,11)$ have been implicated in MHY218-induced apoptotic cell death. In addition, this synthetic hydroxamic acid derivative induces G2/M phase arrest in cells, and this is most likely mediated through the regulation of cyclin $\mathrm{B} 1$ and its activating partners $\mathrm{Cdc} 25 \mathrm{C}$ and Cdc2, and a G2/M phase inhibitor p21 $1^{\text {WAFI/CIP1 }}$ (10-12). MHY218 has been shown to significantly inhibit the growth of tumors in mice bearing human ovarian cancer (10). A recent study has indicated that MHY218 can suppress tamoxifen-resistant breast cancer growth in vivo through the downregulation of the proliferating cell nuclear antigen (PCNA), the induction of apoptotic cell death, and the upregulation of light-chain 3 (LC3), which is a biomarker for autophagy $(10,11)$. Notably, MHY218 possesses more potent anticancer effects compared to those of suberoylanilide hydroxamic acid (SAHA, which is also known as vorinostat) against both ovarian and breast cancer in vivo animal models $(10,11)$.

The aim of the present study was to determine whether MHY218 can induce apoptotic cell death and autophagy in AGS cells, and finally to access the inhibition of autophagy can potentiate the proapoptotic effect of MHY218 in AGS cells.

\section{Materials and methods}

Chemicals. The structure of MHY218 [ $\mathrm{N}^{1}$-hydroxy- $\mathrm{N}^{8}-(4-$ phenoxyphenol)octanedianide] that was used in this study is shown in Fig. 1. This compound was kindly provided by Professor Hyung Ryong Moon (Pusan National University, Busan, Korea), dissolved in dimethyl sulfoxide (DMSO), and stored at $-20^{\circ} \mathrm{C}$ before the experiments, and dilutions were made in culture medium. The maximum concentration of DMSO did not exceed $0.1 \%(\mathrm{v} / \mathrm{v})$ in the treatment range in which there was no influence on cell growth. The DMSO and 3-(4,5-dimethylthiazol-2-yl)-2,5-diphenyl tetrazolium bromide (MTT) were obtained from AMRESCO LLC (Solon, $\mathrm{OH}, \mathrm{USA})$. Antibodies that were specific for procaspase-3, -8 , and -9 , poly(ADP-ribose) polymerase (PARP), Bax, Bcl-2, Beclin-1, and Z-VAD-FMK were obtained from Santa Cruz Biotechnology, Inc. (Dallas, TX, USA). The polyclonal antibody against LC3B was obtained from Cell Signaling Technology, Inc. (Danvers, MA, USA). Propidium iodide (PI), acridine orange, 3-methyladenine, chloroquine (CQ), bafilomycin A1 (Baf-A1), cycloheximide (CHX), and a monoclonal antibody against $\beta$-actin were purchased from Sigma-Aldrich Co. LLC (St. Louis, MO, USA).

Cell culture and cell viability assay. The human GC AGS cell line was cultured in RPMI-1640 (GE Healthcare Life Sciences, Logan, UT, USA) that was supplemented with $10 \%$ fetal bovine serum (GE Healthcare Life Sciences), $100 \mathrm{U} / \mathrm{ml}$ of penicillin and $100 \mu \mathrm{g} / \mathrm{ml}$ of streptomycin (GE Healthcare Life Sciences) at $37^{\circ} \mathrm{C}$ in a humidified $5 \% \mathrm{CO}_{2}$. Cell viability was determined with an MTT assay. For the MTT assay, AGS cells were seeded in a 24-well culture plate, cultured for 24 or $48 \mathrm{~h}$ in the growth media, and then treated with or without various reagents for the indicated concentrations. The cells were incubated in the dark with $0.5 \mathrm{mg} / \mathrm{ml} \mathrm{MTT}$ at $37^{\circ} \mathrm{C}$ for $2 \mathrm{~h}$. The formazan granules that were generated by the live cells were dissolved in DMSO, and the absorbance at $540 \mathrm{~nm}$ was monitored with a multiwell reader (Thermo Fisher Scientific Inc., Vantaa, Finland).

Nuclear staining with Hoechst 33342. Cells were stained with $4 \mu \mathrm{g} / \mathrm{ml}$ of Hoechst 33342 (Life Technologies Corp., Grand Island, NY, USA) at $37^{\circ} \mathrm{C}$ for $10 \mathrm{~min}$. The cells were then examined under a fluorescence microscope.

Measurement of apoptotic cell death by flow cytometry. Apoptotic cell death was determined with Annexin V/PI staining and sub-G1 phase analyses. For the Annexin V/PI staining, the cells were treated with the appropriate conditions for $24 \mathrm{~h}$, subsequently harvested, trypsinized, washed once with cold phosphate-buffered saline (PBS), and then suspended in $1 \mathrm{X}$ binding buffer (BD Biosciences, San Jose, CA, USA). The cells were stained in PI and Annexin V-fluorescein isothio- 


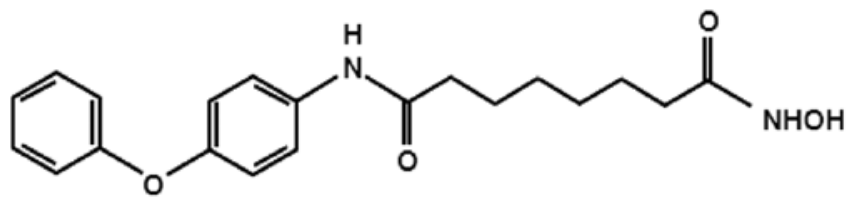

Figure 1.Chemical structure of MHY218 [N1-hydroxy-N8-(4-phenoxyphenol) octanedianide].

cyanate (FITC) solution (BD Pharmingen FITC Annexin V Apoptosis Detection kit) at room temperature for $15 \mathrm{~min}$ in the dark. The stained cells were analyzed by flow cytometry within $1 \mathrm{~h}$. For the sub-G1 phase analysis, the cells were trypsinized, washed once with cold PBS, and then fixed in $70 \%$ ethanol at $-20^{\circ} \mathrm{C}$ overnight. The fixed cells were stained with cold PI solution (50 $\mu \mathrm{g} / \mathrm{ml}$ in PBS) at $37^{\circ} \mathrm{C}$ for $30 \mathrm{~min}$ in the dark. The flow cytometry analysis was performed on Accuri C6 (BD Biosciences).

DNA fragmentation assay. The cells were lysed in buffer containing $5 \mathrm{mM}$ Tris- $\mathrm{HCl}(\mathrm{pH} 7.5), 5 \mathrm{mM}$ EDTA, and $0.5 \%$ Triton X-100 for $30 \mathrm{~min}$ on ice. The lysates were vortexed and cleared by centrifugation at $27,000 \mathrm{x}$ g for $20 \mathrm{~min}$. Fragmented DNA in the supernatant was treated with RNase, which was followed by proteinase $\mathrm{K}$ digestion, phenol/chloroform/ isoamyl alcohol mixture (25:24:1, v/v/v) extraction, and isopropanol precipitation. The DNA was separated in a $1.6 \%$ agarose gel, stained with $0.1 \mu \mathrm{g} / \mathrm{ml}$ ethidium bromide, and visualized with an ultraviolet source.

Western blot analysis. The total cells were lysed in lysis buffer [25 mM Tris (pH 7.5), $250 \mathrm{mM} \mathrm{NaCl}, 5 \mathrm{mM}$ EDTA, $1 \%$ Nonidet $\mathrm{P}-40,100 \mu \mathrm{g} / \mathrm{ml}$ phenymethylsulfonyl fluoride, and protease inhibitor cocktail (Sigma-Aldrich Co. LLC)]. Equal amounts of the protein extracts were denatured by boiling at $100^{\circ} \mathrm{C}$ for 5 min in sample buffer (Bio-Rad Laboratories, Inc., Hercules, CA, USA). The total proteins were subjected to $6-15 \%$ sodium dodecyl sulfate-polyacrylamide gel electrophoresis and transferred to polyvinylidene fluoride membranes. The membranes were probed with the desired primary antibodies overnight, incubated with horseradish peroxidase-conjugated secondary antibodies (Santa Cruz Biotechnology, Inc.), and then visualized with the enhanced chemiluminescence (ECL) detection system (GE Healthcare, Piscataway, NJ, USA).

Caspase activity. The cells were harvested and washed with cold PBS. The total cells were incubated with the lysis buffer (R\&D Systems, Inc., Minneapolis, MN, USA) on ice for $10 \mathrm{~min}$. The lysed cells were centrifuged at $10,000 \mathrm{x} \mathrm{g}$ for $1 \mathrm{~min}$, and $100 \mu \mathrm{g}$ of protein was incubated with $2 \mathrm{X}$ reaction buffer and substrates of colorimetric tetrapeptides, including Z-DEVD for caspase-3, Z-IETD for caspase-8, and Ac-LEHD for caspase-9, respectively. The reaction mixture was incubated at $37^{\circ} \mathrm{C}$ for $2 \mathrm{~h}$, and the enzyme-catalyzed release of p-nitroaniline was quantified at $405 \mathrm{~nm}$ with a multiwell reader (Thermo Fisher Scientific Inc.).

Detection of acidic vesicular organelles (AVOs). The formation of AVOs is a well-known feature of autophagy. Cells were

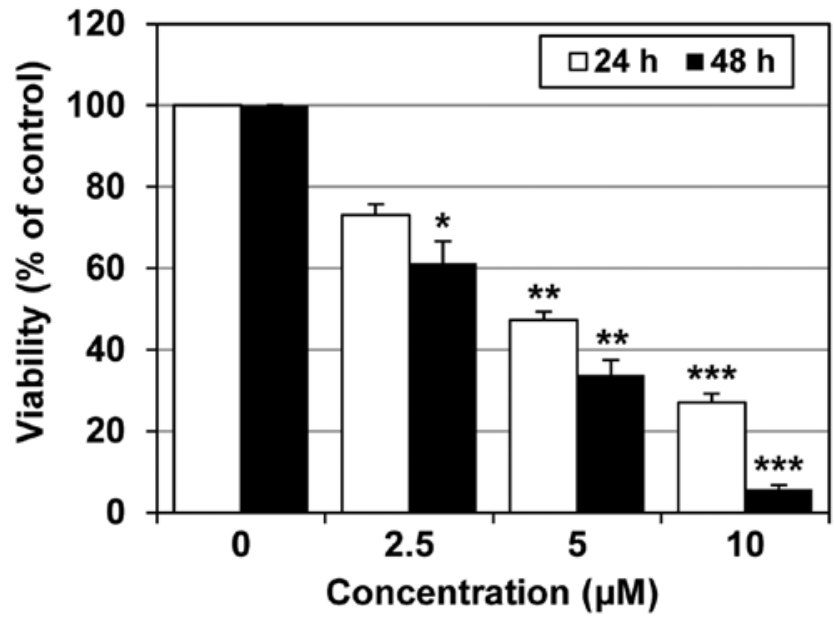

Figure 2. Effects of MHY218 on the viability of AGS cells. The cells were treated with the indicated concentrations of MHY218 for $24 \mathrm{~h}$. Proliferation was determined with a 3-(4,5-dimethylthiazol-2-yl)-2,5-diphenyl tetrazolium bromide (MTT) assay. The results are expressed as mean \pm standard deviation $(\mathrm{SD} ; \mathrm{n}=3)$ and as a percentage of the vehicle-treated control. The significance was determined by Student's t-tests and ANOVA. ${ }^{*} \mathrm{p}<0.05,{ }^{* * *} \mathrm{p}<0.01$ and ${ }^{* * *} \mathrm{p}<0.001$ compared with the vehicle-treated control cells.

treated under the appropriate conditions for $24 \mathrm{~h}$, stained with acridine orange $(1 \mu \mathrm{g} / \mathrm{ml})$ for $15 \mathrm{~min}$, trypsinized, and then washed with PBS. The stained cells were then analyzed with an Accuri C6 flow cytometer (BD Biosciences).

GFP-LC3 assay. Cells were transfected with the LC3-GFP plasmid with the Lipofectamine 2000 reagent (Life Technologies Corp.) according to the manufacturer's protocol. The cells were seeded in Lab-Tek II chamber slides (Thermo Fisher Scientific Inc.). After transfection for $24 \mathrm{~h}$, the cells were treated with $5 \mu \mathrm{M}$ of MHY218 for $24 \mathrm{~h}$, washed with PBS twice, and then fixed with $4 \%$ paraformaldehyde for $20 \mathrm{~min}$. The formation of punctate LC3-positive structures was examined with confocal microscopy. Confocal images were obtained with a FV10i FluoView Confocal Microscope (Olympus Corp., Tokyo, Japan).

Statistical analysis. The results are expressed as the mean \pm standard deviation of three separate experiments, and they were analyzed by Student's t-tests and ANOVA. The means were considered significantly different at ${ }^{*} \mathrm{p}<0.05,{ }^{* *} \mathrm{p}<0.01$ or ${ }^{* * *} \mathrm{p}<0.001$.

\section{Results}

MHY218 exhibited a potent cytotoxic effect on AGS cells. In order to determine whether MHY218 inhibited the growth and proliferation of AGS cells in vitro, we treated the cells for 24 or $48 \mathrm{~h}$ with increasing concentrations of MHY218 that ranged from 0 to $10 \mu \mathrm{M}$. An MTT assay was conducted to measure the MHY218-mediated growth inhibition and estimate the concentration of the compound at which cell growth was cut in half $\left(\mathrm{IC}_{50}\right)$. As shown in Fig. 2, MHY218 effectively suppressed the cell growth of AGS cells in a concentrationdependent manner. Moreover, the $\mathrm{IC}_{50}$ value of MHY218 was $\sim 5$ and $3 \mu \mathrm{M}$ at 24 and $48 \mathrm{~h}$, respectively. The results presented 


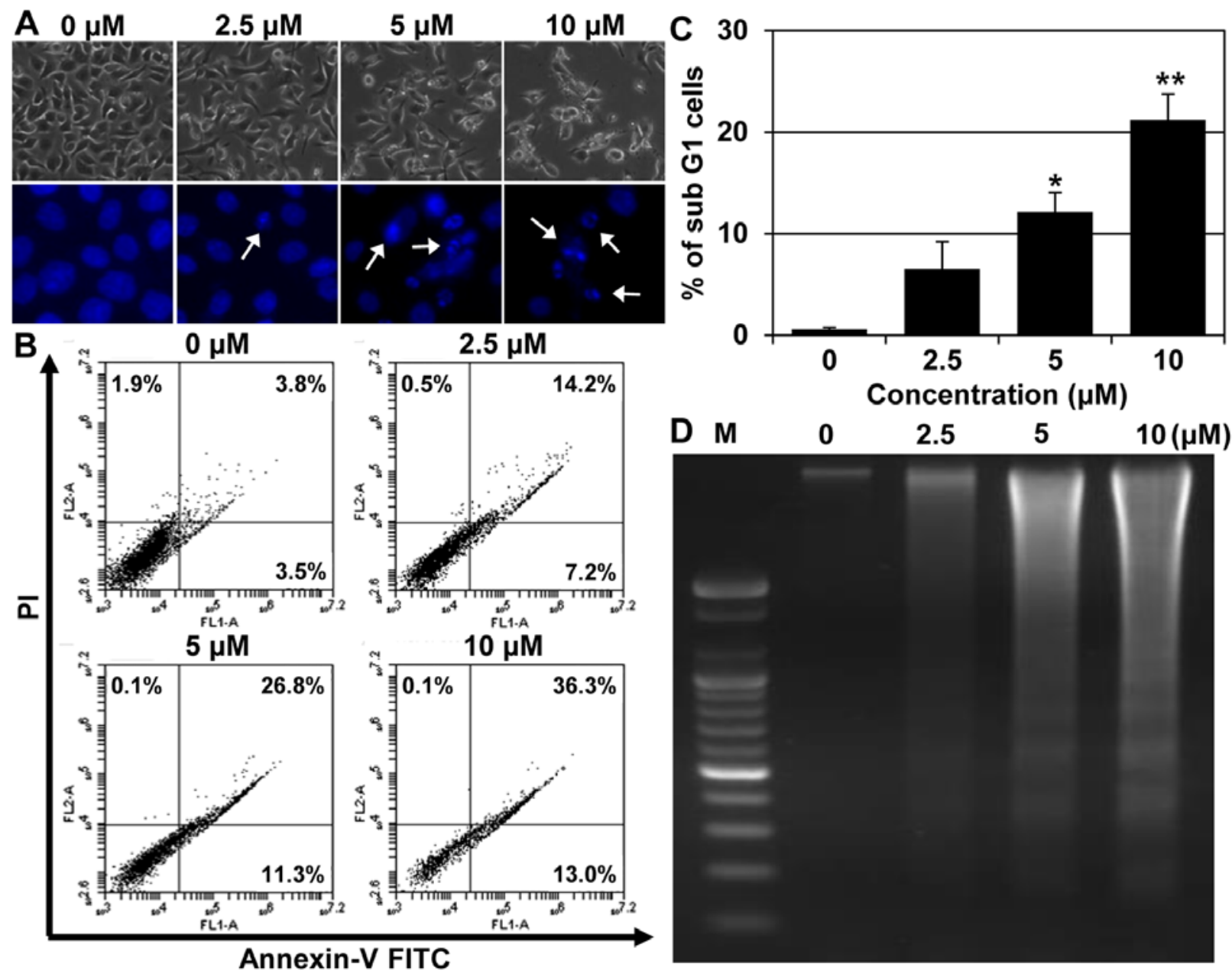

Figure 3. Induction of apoptosis in AGS cells by MHY218. (A) Morphological changes were observed with phase contrast microscopy. The nuclei of AGS cells were stained with the fluorescent DNA-binding dye, Hoechst 33342, and they were then photographed with a fluorescent microscope with a blue filter at a magnification of $\mathrm{x} 400$. Arrows, apoptotic cells. (B) Annexin V-fluorescein isothiocyanate (FITC) binding and propidium iodide (PI) uptake in nonpermeabilized cells were analyzed by flow cytometry. (C) The presence of cells with sub-G1 DNA content following treatment with MHY218 was evaluated with flow cytometry in order to quantify the onset of apoptosis. (D) DNA was extracted and analyzed by $1.6 \%$ agarose gel electrophoresis in the presence of ethidium bromide $(\mathrm{EtBr})$. A representative result is shown from three independent experiments. M, marker.

in Fig. 2 indicate that MHY218 suppressed the growth of AGS cells in a concentration- and time-dependent manner.

MHY218 triggers apoptotic cell death in AGS cells. In order to address the question of whether the growth-suppressive effects of MHY218 were associated with apoptotic cell death, the morphological changes of the cellular structures were assessed with Hoechst 33342 staining. Fig. 3A shows the morphological and nuclear changes in AGS cells after $24 \mathrm{~h}$ of MHY218 treatment. MHY218 treatment caused nuclear condensation and cell death, whereas untreated control cells displayed intact nuclear structures (Fig. 3A). The number of cells with highly condensed nuclei (representing programmed cell death) significantly increased in cells treated with $10 \mu \mathrm{M}$ of MHY218 (Fig. 3A). MHY218-induced apoptotic cell death in AGS cells was further verified with Annexin V and PI double staining. As shown in Fig. 3B, there was a prominent increase in the percentage of late apoptotic cells (36.3\%, with higher Annexin V and PI-positive signals) in the cells after they were treated with $10 \mu \mathrm{M}$ of MHY218 for $24 \mathrm{~h}$ compared to the untreated controls $(0 \mu \mathrm{M}, 3.8 \%)$. Cells with higher
Annexin V but lower PI signals were indicative of early apoptotic cell death. The percentages of total apoptotic cells (early and late apoptotic) significantly increased in AGS cells in a concentration-dependent manner (Fig. 3B). In addition, we examined MHY218-induced cell death in AGS cells with a cell cycle analysis. As shown in Fig. 3C, a cell cycle assay showed that MHY218 significantly increased AGS cells in the sub-G1 population in a concentration-dependent manner (Fig. 3C).

Finally, we examined the effects of MHY218 on DNA fragmentation. AGS cells were treated with various concentrations of MHY218, and inter-nucleosomal DNA fragmentation was evaluated with a DNA ladder on agarose gel electrophoresis. The genomic DNA of the cells treated with MHY218 for $24 \mathrm{~h}$ displayed the characteristic ladder pattern of discontinuous DNA fragments, while untreated cells did not show any signs of fragmentation, as shown in Fig. 3D.

MHY218 modulates the expression of caspases, Bax, and Bcl-2 in AGS cells. The molecular events underlying the MHY218-induced apoptosis in AGS cells were investigated. 


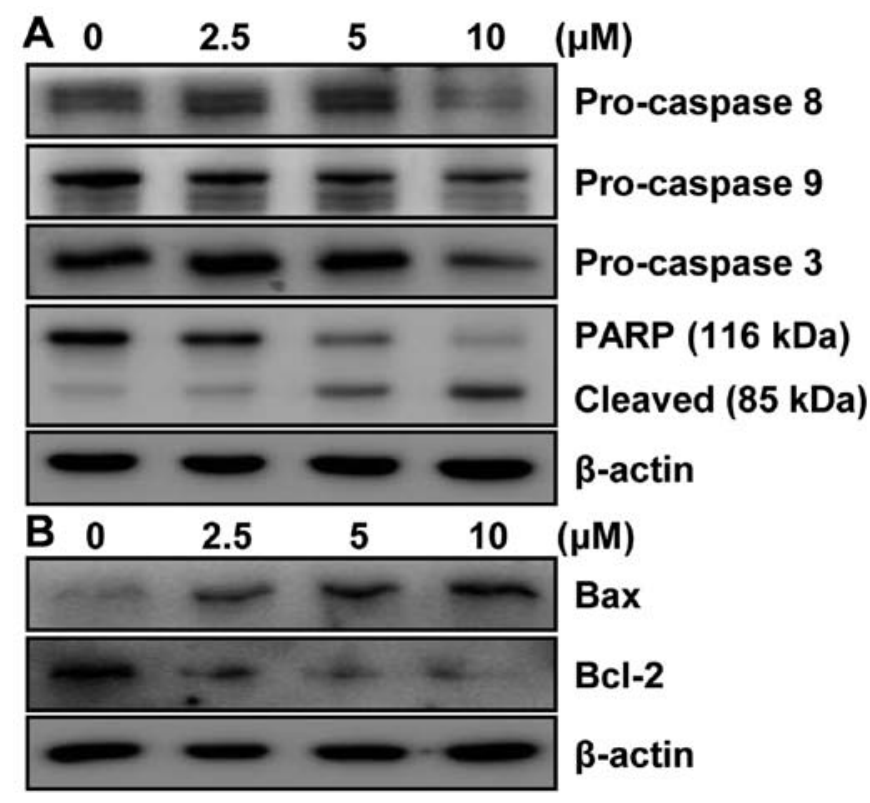

Figure 4. Effects of MHY218 on apoptosis-related protein expression in AGS cells. The cells were treated with variable concentrations of MHY218 for $24 \mathrm{~h}$. (A) Membranes were probed with pro-caspase- $8,-9$, and -3 and poly(ADPribose) polymerase (PARP). (B) The membranes were detected with antibodies against Bax and Bcl-2, and the proteins were visualized with the ECL detection system. Representative results from three independent experiments are shown. Actin was used as a loading control.

The activations of several caspases are important in apoptosis that is induced by various apoptotic stimuli (13). In order to elucidate the mechanisms of MHY218-induced apoptotic cell death in AGS cells, caspase involvement was investigated with a western blot analysis. As shown in Fig. 4A, the exposure of AGS cells to MHY218 markedly decreased the protein levels of pro-caspase-8, -9 and -3 in a concentration-dependent manner. Thus, after treatment with MHY218, the full-length form of the PARP protein $(116 \mathrm{kDa})$, which is a selective substrate for caspase-3, was degraded to the cleaved form (85 kDa) (Fig. 4A).

We next examined the expression of apoptosis-associated proteins after treatment with MHY218. With the balance of anti- and pro-apoptotic proteins arbitrating life-or-death decisions, the $\mathrm{Bcl}-2$ family proteins may regulate mitochondria-dependent apoptosis (14). The expression of pro-apoptotic Bax was increased, while anti-apoptotic Bcl-2 expression was reduced in MHY218-treated cells in a concentration-dependent manner (Fig. 4B). These results suggested that MHY218 promoted cell death in AGS cells through apoptosis with both the intrinsic and extrinsic pathways.

MHY218 induces apoptosis through caspase activation. Because the activation of caspases plays a critical role in apoptosis (15), we examined the activation of caspases by MHY218 in AGS cells. Therefore, the activities of caspases in AGS cells that were treated with various concentration of MHY218 were investigated. After $24 \mathrm{~h}$ of treatment with MHY218, the activities of caspase- $8,-9$, and -3 were increased in a concentration-dependent manner (Fig. 5A). MHY218 markedly stimulated caspase-3 to a $>2$-fold increase in activity
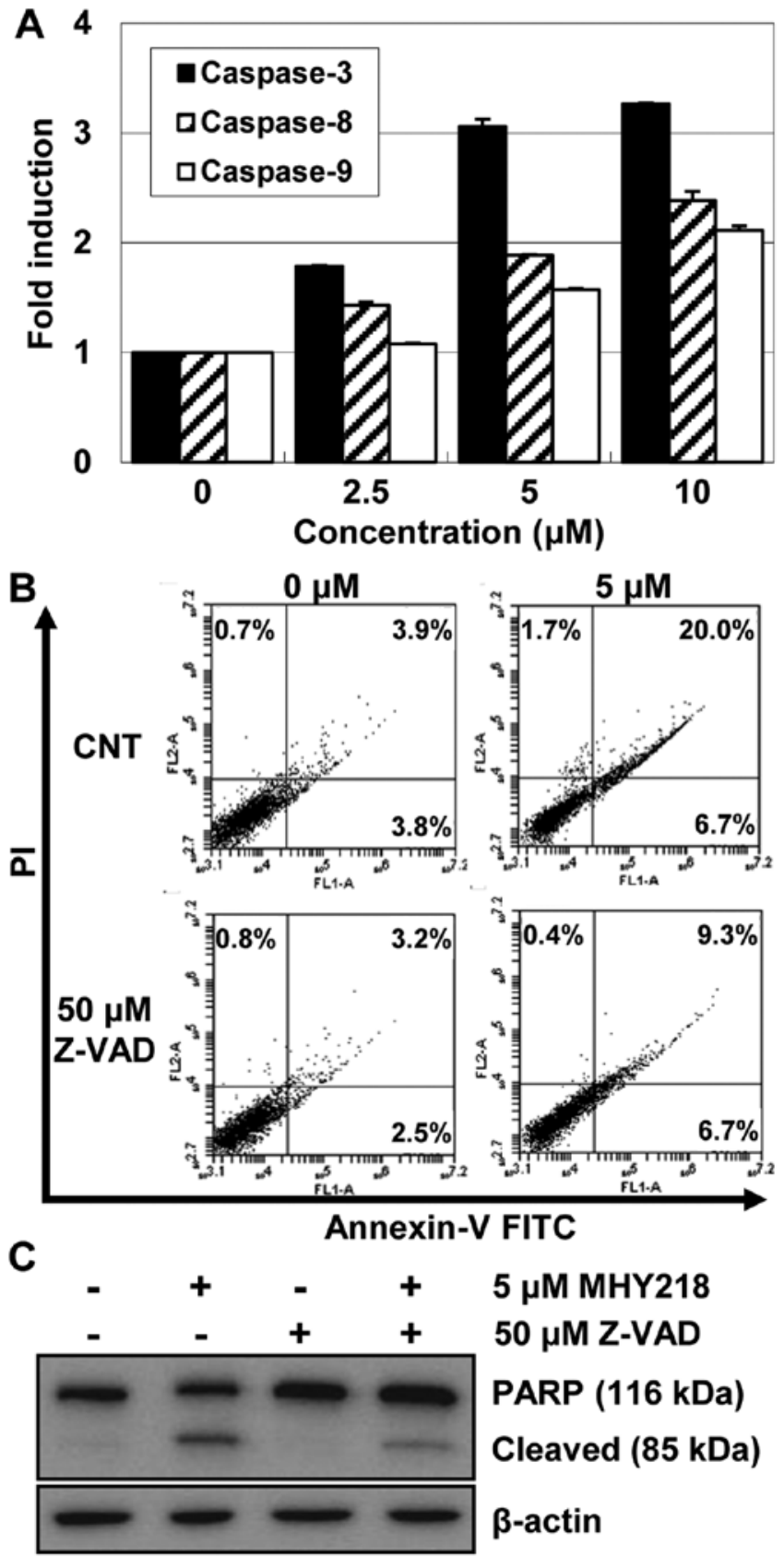

Figure 5. Effects of MHY218 on caspase activation in AGS cells. (A) Cell lysates from cells that were treated with the indicated concentrations of MHY218 for $24 \mathrm{~h}$ were assayed for in vitro caspase-3, -8 , and -9 activity with the substrates of Z-DEVD-pNA, Z-IETD-pNA, and Ac-LEHD-pNA, respectively, at $37^{\circ} \mathrm{C}$ for $2 \mathrm{~h}$. The fluorescent products that were released were measured. The data represent the mean \pm SD values of duplicate samples. The results are representative of one of three independent experiments. (B) The cells were pretreated with $50 \mu \mathrm{M}$ of Z-VAD-FMK for $1 \mathrm{~h}$, and they were then treated with $5 \mu \mathrm{M}$ of MHY218 for $24 \mathrm{~h}$. The cells were stained with Annexin V-FITC/PI and analyzed with flow cytometry. (C) Total cell lysates were prepared and immunoblotted for PARP. The representative results from three independent experiments are shown. Actin was used as a loading control.

in a concentration-dependent manner, while it produced less of an effect on the caspase- 8 and -9 activities.

In order to demonstrate the contributions of caspases to MHY218-induced apoptosis, we next examined the effects of the caspase inhibitor Z-VAD-FMK on apoptosis. As shown in Fig. 5B, Z-VAD-FMK, which is a broad-spectrum caspase 


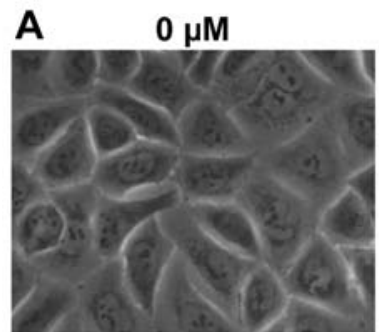

$5 \mu \mathrm{M}$

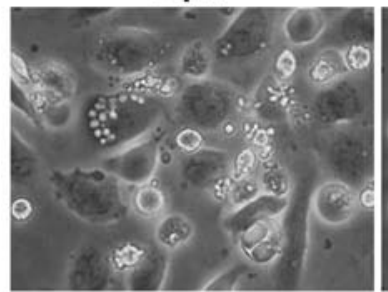

B

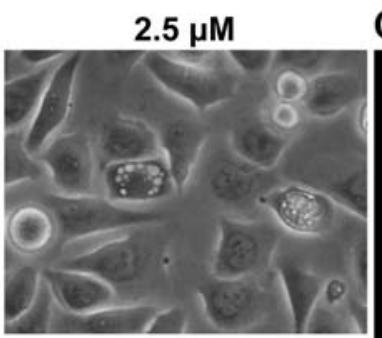

$10 \mu \mathrm{M}$
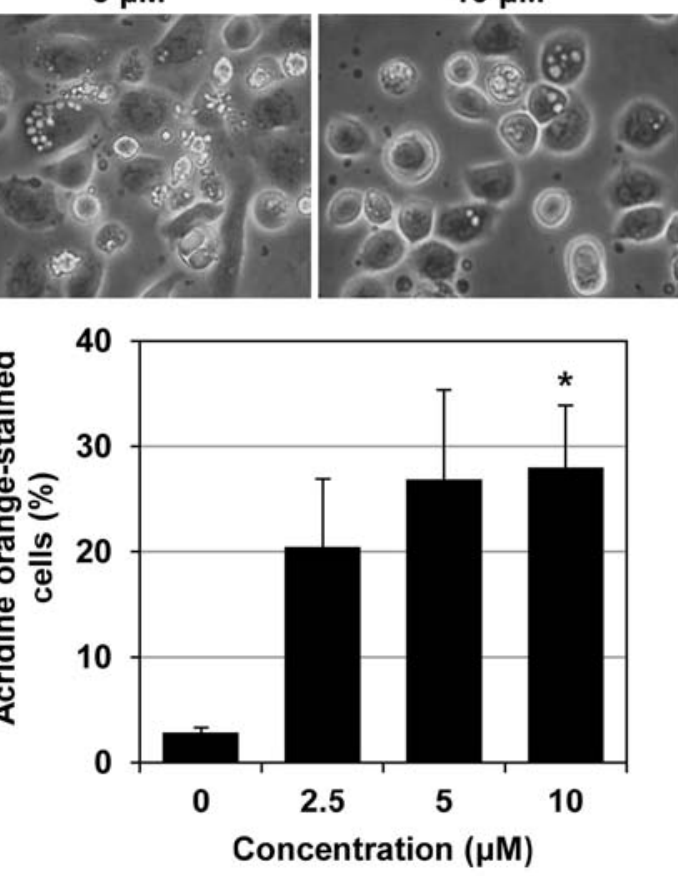

C $\quad 0 \mu \mathrm{M}$
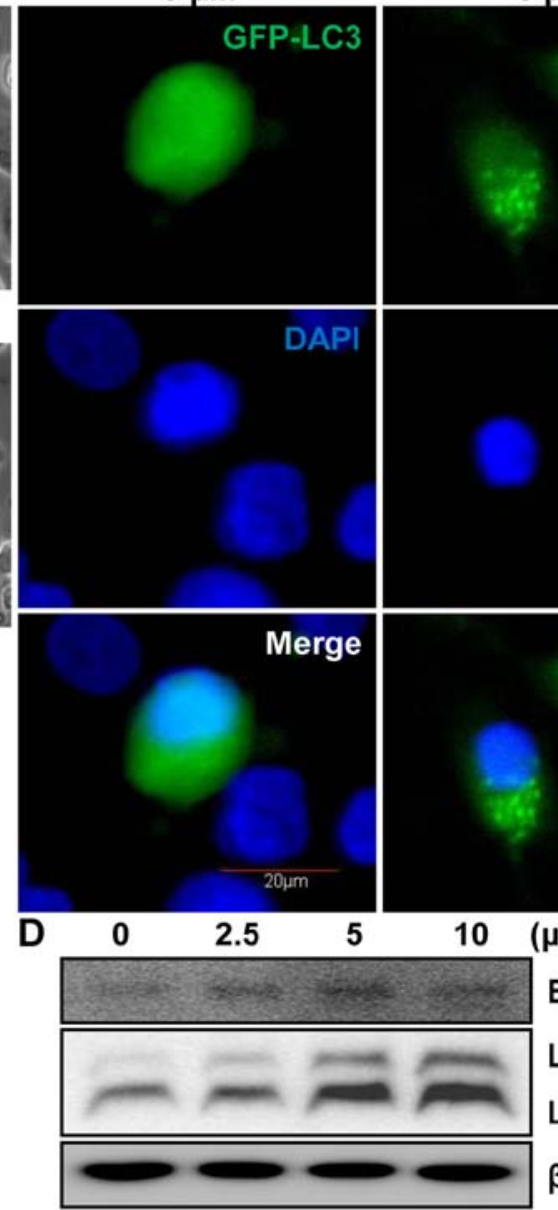

$5 \mu \mathrm{M}$
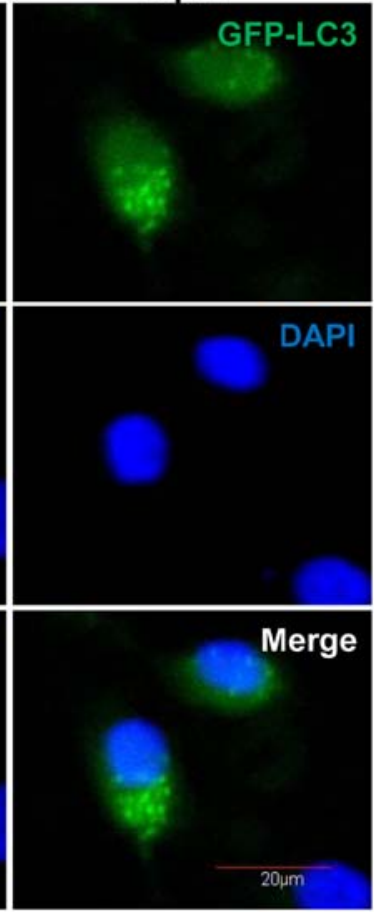

( $\mu \mathrm{M})$

Beclin-1

LC3-I

LC3-II

$\beta$-actin

Figure 6. Induction of autophagy in AGS cells by MHY218. (A) AGS cells were treated with different concentration of MHY218 for 24 h. Representative images were obtained by phase contrast microscopy at a magnification of x400. (B) Cells were treated with MHY218 for $24 \mathrm{~h}$, stained with acridine orange, and then analyzed by flow cytometry to quantify acidic vesicular organelle (AVO) formation. The results are expressed as mean \pm SD (n=3). Significance was determined by Student's t-tests ( ${ }^{*} \mathrm{p}<0.05$ vs. vehicle-treated control cells). (C) AGS cells were transfected with the light-chain 3 (LC3)-green fluorescent protein (GFP) plasmid for $24 \mathrm{~h}$, and the medium was then replaced with media containing $5 \mu \mathrm{M}$ of MHY 218 and incubated for another $24 \mathrm{~h}$. The GFP-LC3 puncta formation was observed with confocal microscopy. The representative results from three independent experiments are shown. Bar, $20 \mu \mathrm{m}$. (D) The cells were lysed, and the levels of Beclin-1 and LC3 proteins were analyzed by western blot analysis.

inhibitor, markedly decreased the proportion of apoptotic cells from 26.7 to $16 \%$, which was the percentage observed in untreated cells. In addition, the effect of the caspase inhibitor on the MHY218-induced cleavage of PARP was examined with a western blot analysis. As shown in Fig. 5C, pretreatment with Z-VAD-FMK inhibited the cleavage of PARP compared to the cells that were treated with MHY218 alone, but not completely. It is interesting to note that MHY218-induced apoptosis was only partially suppressed by Z-VAD-FMK. Taken together, these results suggested that the apoptogenic effect of MHY218 in AGC cells, at least in part, was mediated by activating the caspase cascade.

MHY218 induces autophagy in AGS cells. In recent years, many modes of cell death other than apoptosis have been found to exist, and these include autophagy, necroptosis, and PARP1-mediated cell death (16). In the present study, we examined whether MHY218 also induced autophagy in AGS cells. Cells were treated with the indicated concentrations of MHY218 for $24 \mathrm{~h}$, and the formation of autophagic vacuoles was examined with phase contrast microscopy. As shown in Fig. 6A, MHY218 induced the formation of autophagic vacuoles in AGS cells in a concentration-dependent manner.

We further conducted a series of experiments to confirm the effects of MHY218 on the autophagy process. Because the formation of cytosolic AVOs is one of the typical features of autophagic cell death, a flow cytometry analysis was performed after staining the cells with acridine orange for the quantification of the AVOs. The number of AVOs in MHY218-treated cells clearly increased in a concentration-dependent manner (Fig. 6B).

Beclin-1 levels and the conversion of microtubuleassociated protein $1 \mathrm{LC} 3$ are selective autophagic markers. Next, in order to further confirm that autophagy was induced by MHY218, we examined the LC3 distribution in MHY218treated AGS cells with fluorescence microscopy. As shown in Fig. 6C, green fluorescent protein (GFP)-tagged-LC3 (GFP-LC3) formed cytoplasmic puncta in cells that were treated with MHY218 but not in untreated control cells. In addition, a western blot analysis showed that LC3 underwent a conversion from LC3-I (the soluble form) to LC3-II (the lipidized form) in MHY218-treated cells, thus indicating the 

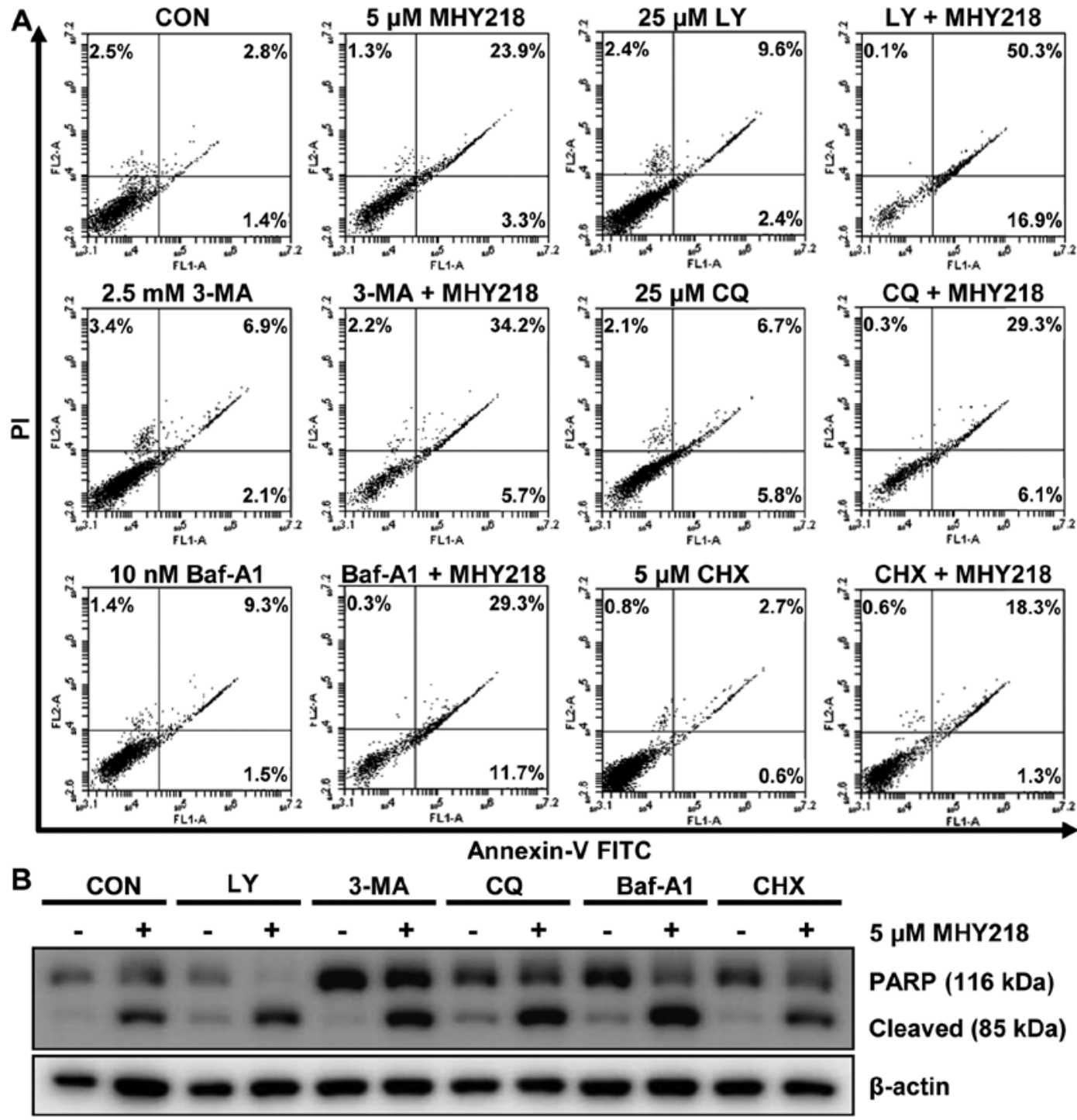

$5 \mu \mathrm{M}$ MHY218

PARP (116 kDa)

Cleaved (85 kDa)

$\beta$-actin

Figure 7. Role of autophagy inhibitors on MHY218-induced apoptosis in AGS cells. (A) AGS cells were pretreated with vehicle, $25 \mu \mathrm{M}$ of LY294002, $2.5 \mathrm{mM}$ of 3-methyladenine (3-MA), $25 \mu \mathrm{M}$ of chloroquine (CQ), $10 \mathrm{nM}$ of bafilomycin A1 (Baf-A1), and $5 \mu \mathrm{M}$ of cycloheximide (CHX) for $1 \mathrm{~h}$, and they were then treated with $5 \mu \mathrm{M}$ of MHY218 for $24 \mathrm{~h}$. Annexin V-FITC binding and PI uptake in non-permeabilized cells were analyzed by flow cytometry. (B) Total proteins were prepared and immunoblotted for PARP. The representative results from three independent experiments are shown. Actin was used as a loading control.

induction of autophagy (Fig. 6D). Consistent with the findings of LC3 conversion, the upregulation of Beclin-1 by MHY218 was observed in AGS cells (Fig. 6D).

The suppression of autophagy modulates MHY218-induced apoptotic cell death in AGS cells. Accumulating data have suggested that the inhibition of autophagy may enhance chemosensitization in human cancer cells (17). Thus, we investigated the role of autophagy in MHY218-induced cell death by examining the effects of pharmacological autophagy inhibitors. Thus, we first treated cells with various autophagy inhibitors for $1 \mathrm{~h}$, incubated them with MHY218 for another $24 \mathrm{~h}$, and then performed Annexin V/PI staining to evaluate cell death. Apoptotic cell death was induced at a level of $27.2 \%$ by MHY218, at a level of $12 \%$ by LY294002, which is an autophagy inhibitor that acts by blocking the class-III phosphoinositide 3-kinases (PI3Ks) that are critical during the late stage of vesicle expansion (autophagosome formation), and at a level of $67.2 \%$ by the combination of the two agents
(Fig. 7A). However, cells that were treated with 3-methyladenine (3-MA), which is an autophagy inhibitor that acts by blocking the class-III PI3Ks, which was followed by the addition of MHY218, exhibited an additive effect on the induction of apoptosis. Similar results were obtained with the use of two autophagy flux blockers, CQ and Baf-A1, on MHY218-induced apoptosis. CQ, which is a lysosomotropic agent, prevents lysosome acidification and thereby the degradation of the products of autophagy, which results in autophagolysosome accumulation, whereas Baf-A1, which is a vacuolar-type $\mathrm{H}^{+}$-ATP inhibitor, prevents the maturation of autophagic vacuoles by inhibiting the fusion of autophagosomes and lysosomes. We also employed CHX, which is an inhibitor of protein biosynthesis and which may inhibit autophagosome formation, to evaluate the effects of MHY218-induced cell death. As shown in Fig. 7A, CHX failed to potentiate the apoptosis-inducing effects of MHY218 in AGS cells, indicating that the effects of the autophagy inhibitor CHX with MHY218 on cell death seemed to be additive. 
To verify the observation that the inhibition of autophagy affected the apoptotic cell death that was induced by MHY218, we performed a western blot analysis to measure PARP cleavage, which is an executioner of apoptosis. MHY218 induced PARP cleavage compared to untreated cells (Fig. 7B). In accordance with the apoptotic cell data (Fig. 7A), cells cotreated with MHY218 and 3-MA, CQ, Baf-A1, or CHX exhibited a measurable increase in PARP cleavage; whereas the combination of the autophagy inhibitor LY294002 and MHY218 resulted in the most profound amounts of PARP cleavage of all of the tested autophagy inhibitors (Fig. 7B). Altogether, these results indicated that the inhibition of autophagy by autophagy inhibitors, such as LY294002, enhanced the MHY218-induced cell death in AGS cells. However, the potency of autophagy inhibition on apoptotic cell death may vary depending on the selectivity of the autophagy inhibitors.

\section{Discussion}

With the development of new therapeutics and the advances in diagnostic technology, the incidence of GC has decreased during the past three decades. However, GC is still the fifth most lethal neoplasm on a worldwide basis, and, thus, Korea continues to have one of the highest rates of GC (18). While surgery is the most common therapy used for patients with stomach cancer, advanced GC (AGC) needs to be treated with multimodal treatments, including chemotherapy and radiotherapy. Thus, the modest efficacies in GC and considerable toxicities that are associated with the current chemotherapy modalities have prompted the pursuit of novel treatment strategies. In this study, we investigated the mechanisms of MHY218, which is a hydroxamic acid derivative, in the induction of autophagy and the role of autophagy in tumor cell survival in the presence of MHY218. Our results indicated that MHY218 triggered both apoptosis and autophagy in AGS cells. Furthermore, we found that autophagy inhibition resulted in higher levels of apoptotic cell death in response to MHY218 treatment. Collectively, our findings suggest that targeting autophagy during cancer treatment with MHY218 may augment the therapeutic effects.

One of the common features of human tumors is the deregulation of HDAC isoenzymes, but no conclusive data about the patterns of HDAC expression in human cancers are available (19-21). Because studies of GC tissues have shown an increase in class-I HDAC expression, HDAC is becoming a prominent therapeutic target for GC treatment (22-24). HDAC inhibitors (HDACi) have been recognized as a new class of therapeutic agents with promising outcomes during the treatment of a broad range of cancer types $(25,26)$. Therefore, HDACi have so far been approved by the US Food and Drug Administration for the treatment of cutaneous $\mathrm{T}$ cell lymphoma, and preclinical and clinical trials have provided evidence that HDACi can be used in the treatment of solid tumors in combination with other anticancer drugs or radiation (26-28). MHY218, which is a synthetic hydroxamic acid, was primarily designed to target HDAC, and it has been shown to suppress HDAC activity and HDAC expression in cancer cells from two solid tumors $(10,11)$. These HDAC expression profiles and previous reports on MHY218 provide the rationale for examining the anticancer potential of MHY218 in GC cells.
Mechanistically, HDACi have been reported to promote apoptosis through alterations in the expression of genes that are involved in apoptotic cell death and activation of the caspase cascade $(29,30)$. We found that MHY218 inhibited cell growth and induced apoptosis in AGS cells. The observed growth-inhibitory and apoptosis-inducing properties of MHY218 were in agreement with those observed by others in ovarian, breast, and colon cancer cells (10-12). When we examined the mechanism by which MHY218 mediated apoptotic cell death in AGS cells, we found that MHY218 activated caspases in AGS cells. In agreement with these observations, previous studies have shown that MHY218 induces caspase activation $(10,12)$. Our observations further revealed that caspase activation in response to MHY218 may be involved in part of the MHY218-induced apoptosis. It is estimated that about half of the induced apoptosis can be inhibited by the pan-caspase inhibitor Z-VAD-FMK, and this is therefore mediated by caspases, whereas the other half is executed in a caspase-independent manner. Likewise, caspase-dependent and -independent apoptosis that is induced by SAHA, which is a widely used HDACi, has also been reported in several cell lines (31-34). Overall, these observations suggest that some other mechanism is likely to be involved in MHY218-induced apoptosis.

We found that MHY218 mediated anticancer activity by modulating the expression of genes that are involved in apoptosis. This hydroxamic acid-derivative treatment resulted in the upregulation of Bax and the downregulation of Bcl-2 expression levels, which was in agreement with the findings of previous reports (10-12). Other HDACi, such as SAHA $(35,36)$, trichostatin A (37), and valproic acid (38), have also been reported to exert apoptosis-inducing effects through the induction of $\mathrm{Bax}$ as well as the inhibition of $\mathrm{Bcl}-2$ expression in cancer cells.

As with many other anticancer therapies, HDACi induces autophagy, and this HDACi-mediated autophagy appears to act as a cytoprotective mechanism to counteract the cytotoxic activities of HDACi $(33,39-41)$. We observed the upregulation of Beclin-1 and an increase in LC3-II conversion in MHY218treated cells. These observations were supported by a previous report that showed that MHY218 induced autophagy in tamoxifen-resistant MCF-7 cells (11). However, Park et al (11) did not rule out the relationship between apoptosis and autophagy that were induced by MHY218 and that cooperate or counteract each other.

Although MHY218 enhanced autophagy, we found that the inhibition of autophagolysosome formation was ineffective on the synergistic induction of apoptosis by MHY218. The inhibition of MHY218-stimulated autophagy by CQ turned out to be ineffective on the synergistic induction of apoptosis by MHY218-CQ cotreatment. This conclusion was further supported by our data that showed that the lysosomal $\mathrm{H}^{+}$-ATPase inhibitor Baf-A1, which similarly interrupted autophagolysosome formation, failed to produce a synergistic effect on MHY218-inducing apoptosis (Fig. 7A). These results indicated that the disruption of autophagolysosome formation per se was not effective on the sensitization of cells for MHY218-mediated apoptosis.

A group of PI3K inhibitors, including 3-MA, wortmannin, and LY294002, have been well established and extensively used 
as autophagy inhibitors, while the inhibition of the PI3K-Akt pathway has previously been reported in certain cancers to trigger autophagy as a survival mechanism (42-44). The effects of PI3K inhibitors as autophagy inhibitors on MHY218-induced apoptosis are intriguing. The combination of MHY218 and the autophagy inhibitor 3-MA enhanced cell death in an additive manner. LY294002, in comparison, dramatically promoted MHY218-induced apoptosis. The cells that were treated with MHY218 exhibited different apoptotic percentages in response to PI3K-targeting autophagy inhibitors. This discrepancy may be explained by the different properties of 3-MA compared to other PI3K inhibitors. A recent report by $\mathrm{Wu}$ and collogues showed a dual role of 3-MA in modulating autophagy (45). They found that, surprisingly, 3-MA promoted autophagy under full (nutrient rich) medium for a prolonged period ( $\leq 9 \mathrm{~h}$ ), whereas it was still capable of inhibiting starvation-induced autophagy. However, the PI3K inhibitor wortmannin was able to suppress autophagy regardless of the nutrient status. An increase in LC3-II levels was observed after $24 \mathrm{~h}$ of treatment with 3-MA (data not shown), even though Wu et al (45) did not determine the LC3 levels with the same experimental period, the possibility that 3-MA activated autophagy as a survival mechanism rather than suppressed MHY218-induced cytoprotective autophagy was proposed. In addition, the effectiveness of the PI3K inhibitors (i.e., 3-MA, wortmannin, and LY294002) was varied in order to induce the autophagic marker LC3-II in different cell types (46), thus further supporting the discrepancy in the results with 3-MA and LY294002 in our present study. Future studies are required to investigate the molecular basis and therapeutic significance of autophagy in the MHY218derived anticancer effects.

In patients with advanced GC, the efficacy of systemic chemotherapy is still limited, and the toxicity of combination chemotherapy is substantial. Hydroxamate-based HDACi, such as vorinostat (SAHA), belinostat (PXD101), and panobinostat (LBH-589), have been shown to exhibit a good toxicity profile, and they are now under phase-I and -II clinical trials in solid tumors with promising results in selected neoplasms, such as hepatocarcinoma (27). A recent phase-I trial of the safety, tolerability, pharmacokinetic, and pharmacodynamic characteristics of hydroxychloroquine (HCQ) in combination with the HDAC inhibitor vorinostat in patients with advanced solid tumors established that $600 \mathrm{mg}$ of HCQ and $400 \mathrm{mg}$ of vorinostat was the maximum tolerated dose and recommended phase-II regimen (47). It is worth noting that the anticancer potency of MHY218 (10 mg/kg, twice a week for 21 days) is greater than that of SAHA $(25 \mathrm{mg} / \mathrm{kg}$, twice a week for 21 days) in tumor-bearing nude mice $(10,11)$. These findings and the promising results from clinical trials encourage further consideration of MHY's ability to be used as a therapeutic target in gastric cancer. However, evaluations in non-human primates of the pharmacokinetics, pharmacodynamics, safety, and efficacy and studies that use clinically relevant animal models to explore more action mechanisms are needed to fully realize the potential of this fascinating molecule in the treatment of gastric cancer.

Altogether, our data suggested that the novel synthetic hydroxamate MHY218 possessed anticancer activity by triggering and autophagy induction in GC cells. The results from the present study provide evidence of MHY218-induced autophagy, and autophagy inhibition by LY294002 dramatically augmented apoptotic cell death that was induced by MHY218. Our findings suggest that MHY218-induced autophagy acts as a prosurvival mechanism against MHY218induced apoptosis.

\section{Acknowledgements}

This study was supported by the National Research Foundation of Korea (NRF) grant funded by the Korea government (MSIP) (no. 2009-0083538). We thank the Aging Tissue Bank for providing research information.

\section{References}

1. Wöhrer SS, Raderer M and Hejna M: Palliative chemotherapy for advanced gastric cancer. Ann Oncol 15: 1585-1595, 2004.

2. Lum JJ, Bauer DE, Kong M, Harris MH, Li C, Lindsten T and Thompson CB: Growth factor regulation of autophagy and cell survival in the absence of apoptosis. Cell 120: 237-248, 2005.

3. Debnath J, Baehrecke EH and Kroemer G: Does autophagy contribute to cell death? Autophagy 1: 66-74, 2005.

4. Levine B and Kroemer G: Autophagy in the pathogenesis of disease. Cell 132: 27-42, 2008.

5. White E and DiPaola RS: The double-edged sword of autophagy modulation in cancer. Clin Cancer Res 15: 5308-5316, 2009.

6. González-Polo RA, Boya P, Pauleau AL, Jalil A, Larochette N, Souquère $S$, Eskelinen EL, Pierron G, Saftig P and Kroemer G: The apoptosis/autophagy paradox: Autophagic vacuolization before apoptotic death. J Cell Sci 118: 3091-3102, 2005.

7. Maiuri MC, Zalckvar E, Kimchi A and Kroemer G: Self-eating and self-killing: Crosstalk between autophagy and apoptosis. Nat Rev Mol Cell Biol 8: 741-752, 2007.

8. Li X and Fan Z: The epidermal growth factor receptor antibody cetuximab induces autophagy in cancer cells by downregulating HIF-1alpha and Bcl-2 and activating the beclin 1/hVps34 complex. Cancer Res 70: 5942-5952, 2010.

9. Amaravadi RK, Yu D, Lum JJ, Bui T, Christophorou MA, Evan GI, Thomas-Tikhonenko A and Thompson CB: Autophagy inhibition enhances therapy-induced apoptosis in a Myc-induced model of lymphoma. J Clin Invest 117: 326-336, 2007.

10. Jeon HS, Ahn MY, Park JH, Kim TH, Chun P, Kim WH, Kim J, Moon HR, Jung JH and Kim HS: Anticancer effects of the MHY218 novel hydroxamic acid-derived histone deacetylase inhibitor in human ovarian cancer cells. Int J Oncol 37: 419-428, 2010.

11. Park JH, Ahn MY, Kim TH, Yoon S, Kang KW, Lee J, Moon HR, Jung JH, Chung HY and Kim HS: A new synthetic HDAC inhibitor, MHY218, induces apoptosis or autophagy-related cell death in tamoxifen-resistant MCF-7 breast cancer cells. Invest New Drugs 30: 1887-1898, 2012.

12. Kim MK, Kang YJ, Kim DH, Hossain MA, Jang JY, Lee SH, Yoon JH, Chun P, Moon HR, Kim HS, et al: A novel hydroxamic acid derivative, MHY218, induces apoptosis and cell cycle arrest through downregulation of NF- $\kappa \mathrm{B}$ in HCT116 human colon cancer cells. Int J Oncol 44: 256-264, 2014.

13. Kumar S: Caspase function in programmed cell death. Cell Death Differ 14: 32-43, 2007.

14. Tsujimoto Y: Role of Bcl-2 family proteins in apoptosis: Apoptosomes or mitochondria? Genes Cells 3: 697-707, 1998.

15. Thornberry NA and Lazebnik Y: Caspases: Enemies within. Science 281: 1312-1316, 1998.

16. Degterev A and Yuan J: Expansion and evolution of cell death programmes. Nat Rev Mol Cell Biol 9: 378-390, 2008.

17. Carew JS, Nawrocki ST and Cleveland JL: Modulating autophagy for therapeutic benefit. Autophagy 3: 464-467, 2007.

18. Jung KW, Won YJ, Kong HJ, Oh CM, Lee DH and Lee JS: Cancer statistics in Korea: Incidence, mortality, survival, and prevalence in 2011. Cancer Res Treat 46: 109-123, 2014.

19. Ropero S and Esteller M: The role of histone deacetylases (HDACs) in human cancer. Mol Oncol 1: 19-25, 2007.

20. Nakagawa M, Oda Y, Eguchi T, Aishima S, Yao T, Hosoi F, Basaki Y, Ono M, Kuwano M, Tanaka M, et al: Expression profile of class I histone deacetylases in human cancer tissues. Oncol Rep 18: 769-774, 2007. 
21. Yoo CB and Jones PA: Epigenetic therapy of cancer: Past, present and future. Nat Rev Drug Discov 5: 37-50, 2006.

22. Choi JH, Kwon HJ, Yoon BI, Kim JH, Han SU, Joo HJ and Kim DY: Expression profile of histone deacetylase 1 in gastric cancer tissues. Jpn J Cancer Res 92: 1300-1304, 2001

23. Song J, Noh JH, Lee JH, Eun JW, Ahn YM, Kim SY, Lee SH, Park WS, Yoo NJ, Lee JY, et al: Increased expression of histone deacetylase 2 is found in human gastric cancer. APMIS 113: 264-268, 2005.

24. Weichert W, Röske A, Gekeler V, Beckers T, Ebert MP, Pross M, Dietel M, Denkert C and Röcken C: Association of patterns of class I histone deacetylase expression with patient prognosis in gastric cancer: A retrospective analysis. Lancet Oncol 9: 139-148, 2008.

25. Khan $\mathrm{O}$ and La Thangue NB: HDAC inhibitors in cancer biology: Emerging mechanisms and clinical applications. Immunol Cell Biol 90: 85-94, 2012.

26. Slingerland M, Guchelaar HJ and Gelderblom H: Histone deacetylase inhibitors: An overview of the clinical studies in solid tumors. Anticancer Drugs 25: 140-149, 2014.

27. Grassadonia A, Cioffi P, Simiele F, Iezzi L, Zilli M and Natoli C: Role of hydroxamate-based histone deacetylase inhibitors (Hb-HDACIs) in the treatment of solid malignancies. Cancers (Basel) 5: 919-942, 2013.

28. Nolan L, Johnson PW, Ganesan A, Packham G and Crabb SJ: Will histone deacetylase inhibitors require combination with other agents to fulfil their therapeutic potential? Br J Cancer 99: 689-694, 2008.

29. Emanuele S, Lauricella M and Tesoriere G: Histone deacetylase inhibitors: Apoptotic effects and clinical implications (Review). Int J Oncol 33: 637-646, 2008.

30. Ellis L and Pili R: Histone deacetylase inhibitors: Advancing therapeutic strategies in hematological and solid malignancies. Pharmaceuticals (Basel) 3: 2411-2469, 2010.

31. Dong G, Wang L, Wang CY, Yang T, Kumar MV and Dong Z: Induction of apoptosis in renal tubular cells by histone deacetylase inhibitors, a family of anticancer agents. J Pharmacol Exp Ther 325: 978-984, 2008.

32. Xu W, Ngo L, Perez G, Dokmanovic M and Marks PA: Intrinsic apoptotic and thioredoxin pathways in human prostate cancer cell response to histone deacetylase inhibitor. Proc Natl Acad Sci USA 103: 15540-15545, 2006.

33. Shao Y, Gao Z, Marks PA and Jiang X: Apoptotic and autophagic cell death induced by histone deacetylase inhibitors. Proc Nat Acad Sci USA 101: 18030-18035, 2004.

34. Hrzenjak A, Kremser ML, Strohmeier B, Moinfar F, Zatloukal K and Denk H: SAHA induces caspase-independent, autophagic cell death of endometrial stromal sarcoma cells by influencing the mTOR pathway. J Pathol 216: 495-504, 2008

35. Wang YC, Kong WZ, Xing LH and Yang X: Effects and mechanism of suberoylanilide hydroxamic acid on the proliferation and apoptosis of human hepatoma cell line Bel-7402. J BUON 19: 698-704, 2014

36. Zhang XD, Gillespie SK, Borrow JM and Hersey P: The histone deacetylase inhibitor suberic bishydroxamate regulates the expression of multiple apoptotic mediators and induces mitochondria-dependent apoptosis of melanoma cells. Mol Cancer Ther 3: 425-435, 2004.
37. Park H, Lee YJ, Kim TH, Lee J, Yoon S, Choi WS, Myung CS and Kim HS: Effects of trichostatin A, a histone deacetylase inhibitor, on the regulation of apoptosis in H-ras-transformed breast epithelial cells. Int J Mol Med 22: 605-611, 2008.

38. Shen WT, Wong TS, Chung WY, et al: Valproic acid inhibits growth, induces apoptosis, and modulates apoptosis-regulatory and differentiation gene expression in human thyroid cancer cells. Surgery 138: 979-985, 2005.

39. Choi EJ, Cho BJ, Lee DJ, Hwang YH, Chun SH, Kim HH and Kim IA: Enhanced cytotoxic effect of radiation and temozolomide in malignant glioma cells: Targeting PI3K-AKT-mTOR signaling, HSP90 and histone deacetylases. BMC Cancer 14: 17, 2014.

40. El-Khoury V, Pierson S, Szwarcbart E, Brons NH, Roland O, Cherrier-De Wilde S, Plawny L, Van Dyck E and Berchem G: Disruption of autophagy by the histone deacetylase inhibitor MGCD0103 and its therapeutic implication in B-cell chronic lymphocytic leukemia. Leukemia 28: 1636-1646, 2014.

41. Watanabe M, Adachi S, Matsubara H, Imai T, Yui Y, Mizushima Y, Hiraumi Y, Watanabe K, Kamitsuji Y, Toyokuni SY, et al: Induction of autophagy in malignant rhabdoid tumor cells by the histone deacetylase inhibitor FK228 through AIF translocation. Int J Cancer 124: 55-67, 2009.

42. Degtyarev M, De Mazière A, Orr C, Lin J, Lee BB, Tien JY, Prior WW, van Dijk S, Wu H, Gray DC, et al: Akt inhibition promotes autophagy and sensitizes PTEN-null tumors to lysosomotropic agents. J Cell Biol 183: 101-116, 2008.

43. Mirzoeva OK, Hann B, Hom YK, Debnath J, Aftab D, Shokat K and Korn WM: Autophagy suppression promotes apoptotic cell death in response to inhibition of the PI3K-mTOR pathway in pancreatic adenocarcinoma. J Mol Med Berl 89: 877-889, 2011.

44. Fan QW, Cheng C, Hackett C, Feldman M, Houseman BT, Nicolaides T, Haas-Kogan D, James CD, Oakes SA, Debnath J, et al: Akt and autophagy cooperate to promote survival of drugresistant glioma. Sci Signal 3: ra81, 2010.

45. Wu YT, Tan HL, Shui G, Bauvy C, Huang Q, Wenk MR, Ong CN, Codogno P and Shen HM: Dual role of 3-methyladenine in modulation of autophagy via different temporal patterns of inhibition on class I and III phosphoinositide 3-kinase. J Biol Chem 285: 10850-10861, 2010.

46. Cheng J, Ohsaki Y, Tauchi-Sato K, Fujita A and Fujimoto T: Cholesterol depletion induces autophagy. Biochem Biophys Res Commun 351: 246-252, 2006.

47. Mahalingam D, Mita M,Sarantopoulos J, Wood L, AmaravadiRK, Davis LE, Mita AC, Curiel TJ, Espitia CM, Nawrocki ST, et al: Combined autophagy and HDAC inhibition: A phase I safety, tolerability, pharmacokinetic, and pharmacodynamic analysis of hydroxychloroquine in combination with the HDAC inhibitor vorinostat in patients with advanced solid tumors. Autophagy 10: 1403-1414, 2014. 